\title{
Property Registration Framework using Ethereum Blockchain
}

\author{
Madhurya J A ${ }^{1}$, Beena G Pillai ${ }^{2}$, Dr.Dayananda Lal ${ }^{3}$, Dr.I Jeena Jacob ${ }^{4}$ \\ ${ }^{1}$ Assistant Professor, Department of CSE, GITAM School of Technology, Bengaluru, India, \\ mambuja@gitam.edu \\ ${ }^{2}$ Assistant Professor, Department of CSE, GITAM School of Technology, Bengaluru, India, bpillai@ gitam.edu \\ ${ }^{3}$ Assistant Professor, Department of CSE, GITAM School of Technology, Bengaluru, India, \\ dnarayan@gitam.edu \\ ${ }^{4}$ Associate Professor, Department of CSE, GITAM School of Technology, Bengaluru, India, ijacob@ gitam.edu
}

\begin{abstract}
The land is an immovable property. Tracing the details of property and involvement of third-party is the challenging task. Fraudulent may forge documents and mislead the purchaser and if it's a litigate land it takes many years in the court battle, waste of time and resource. Problems escalate in this current land registration process which is non-transparent. Such demands made to integrate technologies and lead to the blooming of blockchain technology. Blockchain is a decentralized and transparent ledger. The smart contract is the confirmable and permanent document between two parties. This proposed framework is used to develop a registration application system in which new purchaser has to register and continue with the further process. The proposed research work is implemented using SHA256 algorithm which provides unique hash values for the messages getting stored in blocks and ethereum blockchain technology is used to store both smart contract and transaction details.
\end{abstract}

Key words : Decentralised, ethereum blockchain, land registration platform, smart contract, SHA algorithm.

\section{INTRODUCTION}

From these recent years, blockchain technology is booming, it has significant growth from the past few years. Blockchain technology can be widely used in multiple regions like food chain supplement, land registry, sensor networks and IoT [2]. It is originated from the nucleus of component Bitcoin. Currently, individuals have to carry hard cash or need to do the online transfer which may lead to transaction frauds if a single digit in account number is misplaced. Since a huge amount of data is being exchanged, providing security will be a complex task. This may lead to mistrust, corruption, hacking of data, insecurity and fraudulent. Such demands made to integrate technologies and lead to the blooming of blockchain technology. The key to solving these complexities using blockchain technology is by encrypting the information and thus storing in distributed ledger or data which is decentralized and provides transparency of information[3].

In Blockchain Technology, a digital currency called Bitcoins or Cryptocurrency is used for transactions. Due to the success of digital currency security can be achieved in financial transactions. These technologies and techniques are centralized and owned by authorities.

Every country follows their government rules, to transfer the land or property to other individuals. Now a day's frauds are more in land registration because tracking the details of property like who owns the property, rate of the land, single owned or multi-owned property and does it has any litigate issues all these are difficult tasks. And more importantly, the third party is involved where they may quote a high price or else time-delay in the registration process. So to avoid all issues or frauds we are implementing a land registry platform by using blockchain technology[3].

Here a concept called smart contract is used in blockchain technology which is a self-agreement embedded code in a system. This code contains a certain set of rules to be followed by the involved individuals in the land registration process and further procedure will be continued if smart contract conditions are met. This smart contract will be automatically activated if conditions specified in the contract are met. This will help to eliminate the need of third party person and the registration process will be faster and efficient.

Thus smart contract is placed in a decentralized server. By decentralizing, these contracts are not controlled by any party, but it will be distributed in a shared database run by many systems. Thus none of them will have control over the data, which means it is near impossible to hack it[3]. Suppose the hacker need to misuse the data they need to attack blockchain blocks or smart contract block where it is stored. To get that information they need to access more than half nodes to get information. Smart contracts will then run securely and instantly, without anyone being able to modify them. 
Madhurya J A et al., International Journal of Emerging Trends in Engineering Research, 8(9), September 2020, 5209 - 5213

Along with a smart contract, Ethereum is also used because transactions that occurred as bitcoins details have to be stored securely[4]. So these documents are also stored in ethereum blocks where the smart contract is also stored. Since this is also stored in the decentralized server, security is achieved for the transaction. Ethereum based smart contract is developed using the solidity programming language. SHA256 hash algorithm is used to generate unique hash values for each blocks where all bitcoins transaction details and smart contract is stored.

\section{RELATED WORK}

Xiaolong et.al [1] have described ethereum smart contract, as well as its main equipment function, provided the architecture for ethereum smart contract thus increases the installation cost and its most significant expertise comprises of blockchain process, protection knowledge and explanation and monitors contracts. And data is not limited without any filtering applied on ethereum node completed data can be fetched.

Yining Hu et.al [9] have specified about blockchain and its various applications and characteristics involved in smart contract such as isolation, security, anonymity, collaboration, and untraceability which provide useful reimbursement to a variety of fields. Investigating relevance of blockchain can be applied in health care, identity management, banking sector, authority asset.

Harry Halpin et.al [7] have specified about security and privacy in blockchain. It proposed bitcoin and its variants provides more security when compared to cryptographers. Bitcoin only offers transactions pseudonymised. The regulation of block arrangement and development is projected in the chain, to which a computation depiction is formed. And confidential transfers of bitcoins was suggested to achieve privacy.

Ashwin et.al [8] have described blockchain land registry management, where it would revolutionise the existing structure by simultaneously eradicating existing issues. They have proposed a framework in which encroached chain could be used to protect all property details. They have only considered registration document and khata id in this model and created block to store all these details. But here client itself has to store these entire documents in block that's the main limitation.

Meghali Nandi et.al [3] have proposed about secure record keeping mechanism to solve several property problems using a Blockchain-based system where that will create physical records into a immutable records in Blockchain technology. These new block assets can now be used to preserve a digitally protected record and can access ownership history.

\subsection{Current Challenges}

Nowadays fraud is accomplished in any transaction. Tracking the details of property like who owns the property, rate of the land, single owned or multi-owned property, does it has any litigate issues are all difficult tasks. The challenges that are existing in the present land registry are [10]

1. Involvement of third person where for commission purpose they will quote the high rate or may show fake documents

2. The number of frauds is increasing day by day, where the imposter pretends to be the owner of the property and provides forged documents.

3. Time delays-it may take several months to complete the land registration. Suppose if the land has any litigate issues then that leads to a court battle between parties.

So the buyer's should have much knowledge of market trending and to know the actual rate of the property to avoid all these consequences. But all the individuals don't have complete knowledge of this field that leads to different complications. So here proposed a blockchain technology for land registration, due to its transparent nature easily buyers can trace the property details thus trying to avoid fraudulently.

\section{METHODOLOGY}

The purpose to implement this methodology is to avoid fraudulence in land registration and to secure their transaction details. The main problem in the existing system is purchaser didn't use to get the actual property details due to imposters. So here we are using Ethereum blockchain technology which is transparent, immutable and decentralized[3]. In the blockchain, we are creating a land registry platform where all the details of the property which are earlier registered will be stored on a decentralized database, which is transparent, so any individuals who wish to purchase a property can cross-verify the complete details of the property. Details of each land will be stored in the form of block and digital title will also be given to make search efficient, where for each block a unique hash value will be assigned by using the SHA256 algorithm. Searchers can search the property with digital titles in that land registry platform. Overview of the architecture is shown in Figure.1

Suppose the property is already registered and owned by a particular owner, those details will be stored in registry platform means decentralized database by the land administration person, and it is placed in the form of blocks in the blockchain. Thus admin gives a created land ID to the owner so that a particular owner can access the details of the property[10]. 


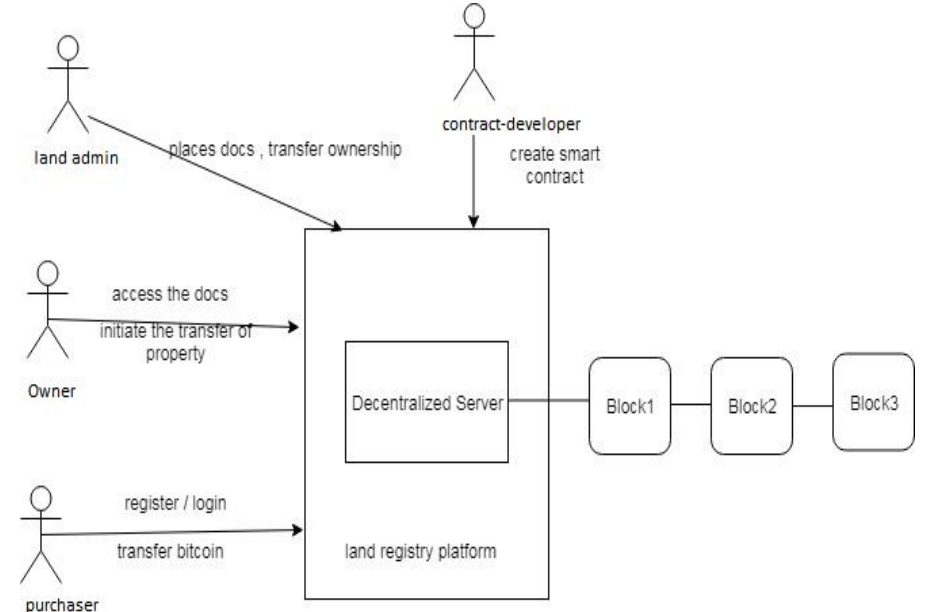

Figure 1: An overview of the proposed architecture

\subsection{Steps}

1. Administrator access to registry platform:

i) The already registered property details like land document, owner name, complete address, from whom they bought, purchased price, loan details if applicable, all these information will be stored on the decentralized server i.e. inside ethereum blockchain, in the form of blocks which has unique hash value, each land details will be stored, and digital title will be given to make search easy. Later admin generates land ID and issues it to the owner for future access.

ii) Once the administrator receives a transfer of property request, they will verify and approve it. Later they will provide the land ID to the new owner and update the new owner details in the block and places land documents in the block, as referred in Figure 2(a).

2. Owner wishes to sell property:

i) The owner accesses their property details by login to the platform by using the land ID. If they wish to sell their property for any reason, they will specify the rules to create a smart contract in the block to the developer and these smart contracts are stored where land documents are already stored by admin. Smart contract means a set of rules will be specified like quoted price etc. if these rules are agreed by the purchaser then-owner initiates transfer of land authority.

ii) Communicate with the owner request received from the purchaser to continue the further proceedings. The owner can approve or reject the request by viewing the complete information of the requested purchaser.

iii) After receiving the quoted price in the form of bitcoins by the purchaser, seller initiates transfer property. This request will be sent to the land administrator referred in Figure 2(b).

3. Contract-developer: person's role is to create smart-contract based on rules specified by the owner of the property and stores this smart contract in ethereum blockchain. The owner can't do any changes directly in the smart contract.

4. Purchaser registers to platform and view property:

i) The purchaser first needs to register for the platform by providing their complete details. After successful registration, they receive a registered ID. Through the ID login into the platform and they can search the required property by specifying digital title. Based on purchaser interest can view the land documents and smart contract, if they are interested to purchase the property, then they need to send a request to communicate to the owner.

ii) After communicating with the owner, if all the terms are agreed upon, then the quoted price will be paid by the purchaser in the form of bitcoin.

To keep all transaction details secure, these transaction details are stored in the same ethereum block where all land documents and smart contract is stored referred in Figure.2(c)

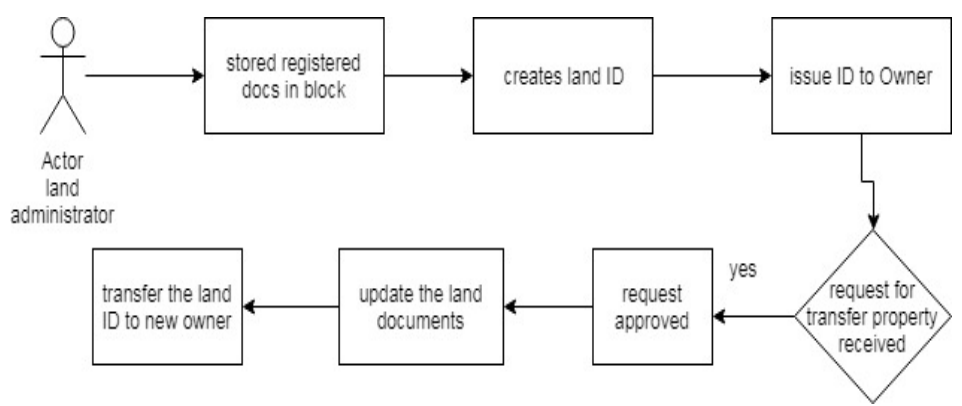

Figure 2(a): Workflow of the land administrator

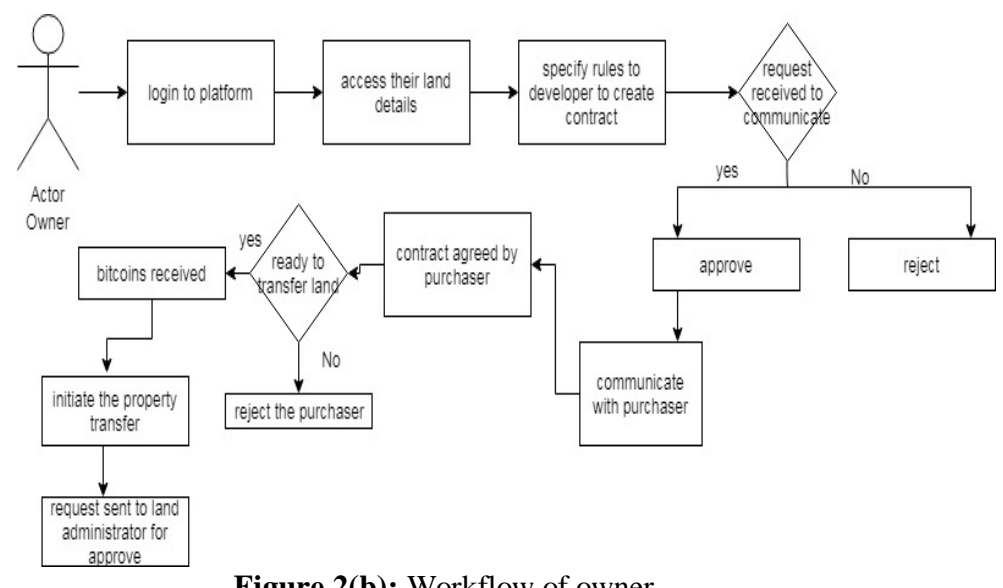

Figure 2(b): Workflow of owner

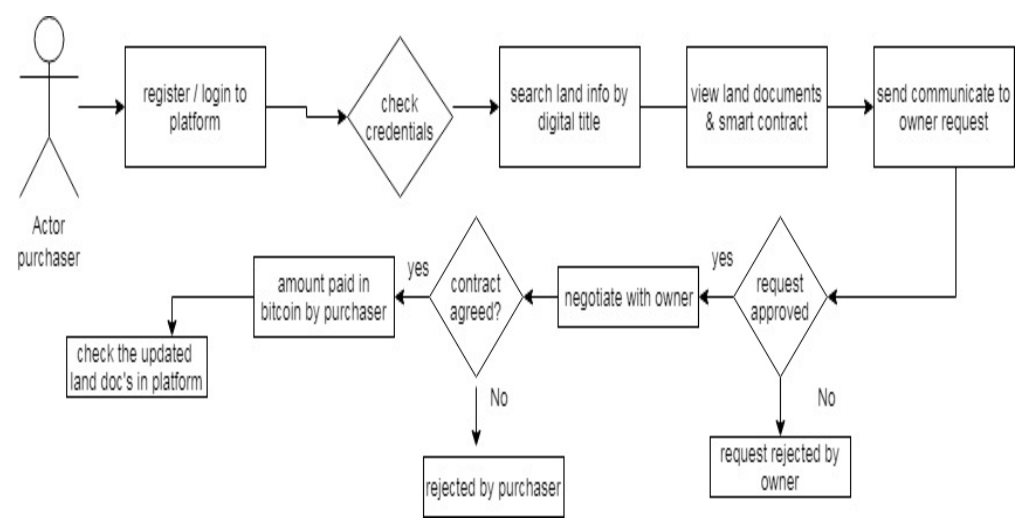

Figure 2(c): Workflow of purchaser 
Madhurya J A et al., International Journal of Emerging Trends in Engineering Research, 8(9), September 2020, 5209 - 5213

Whenever a blockchain is incorporated into a new blockchain transaction or some new block is added to the blockchain means several nodes are required to implement algorithms to evaluate, verify, and process the blockchain context within the same blockchain implementation. The new block of blockchain transaction will be accepted into the ledger when most nodes authenticate the block 's history and signature, and the new block with data will be added to the database. If a consensus is not achieved, they refuse to add the block to the blockchain. This distributed consensus model allows blockchains to operate as a distributed ledger, without any central or unifying authority having to authorise blockchain transactions. So the transaction is highly secure in the blockchain [11].

\section{RESULT}

A new buyer who wishes to purchase land has to register themselves for land registry platform, by providing complete information that has been mentioned in the form. After successful registration particular ID will be given to the new user, as referred in Figure 3(a).

create a new account
First Name:
Last Name:
E-mail:
ph-no:
Country:
Designation:
Company address:
Communication address :
Permanent address:
submit Reset
Figure 3(a): New purchaser's registration form

Administrator after storing all property document in block and then generates unique hash value and adds digital title to this block to make search easier for the property searcher as referred in Figure 3(b).

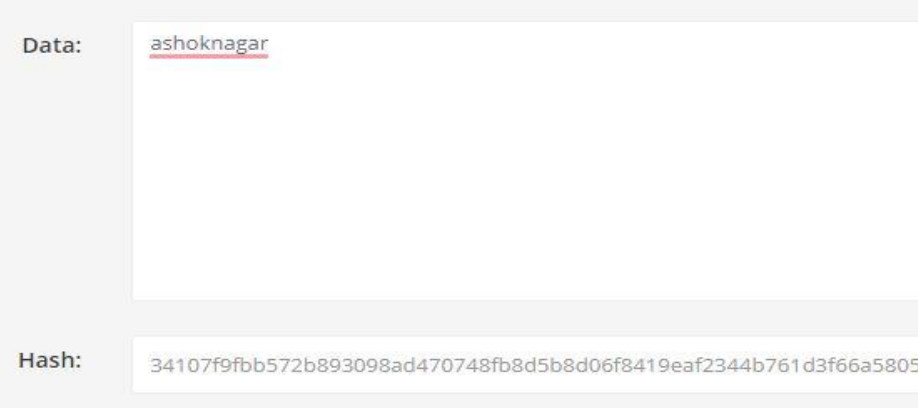

Figure 3(b): Hash value generation
A buyer after getting id, can log in to the portal and perform search operation by specifying the title of the property and sends request access as referred in Figure 3(c).

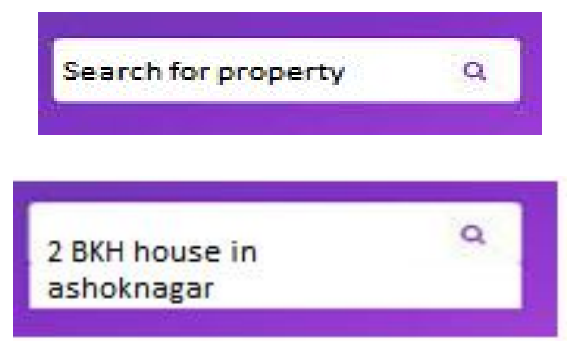

Figure 3(c): Purchaser can search for property

Since blockchain is decentralized, immutable, it reduces the frauds occurring in land registration as third-party is not involved here and the purchaser can view the documents directly. Since documents are uploaded to block by administrator there will be no forged land documents. There is no time delay in the registration process as soon as the quoted price is exchanged in the form of bitcoins, updated registration docs will be updated in a block. These transactions docs are stored on ethereum block where the smart contract is stored, so provides security to the data.

\section{CONCLUSION}

With the use of smart contract in blockchain technology, worldwide transactions are occurred in more secure viewpoint, because all transaction details are stored in the decentralized server, means a data is stored in multiple nodes, where misuse of information is nearly impossible. Ethereum block is used to store all transactions details which occurred in the form of bitcoins and also smart contract. Third-party involvement is completed avoided and there will be no forged documents since the administrator is going to upload all registered documents. A registered purchaser can easily know the complete information of the property and does this land has any litigate issues, so frauds occurring can be avoided and security is achieved.

\section{REFERENCES}

1. Xiaolong Liu, Riqing Chen, Yu-Wen Chen, Shyan-Ming Yuan "Off-chain Data Fetching Architecture for Ethereum Smart Contract", Digital Object Identifier 10.1109/ACCESS.2018.2874539 VOLUME 6, 2018

2. Joe AbouJaoudeAndRaafat George Saade, "Blockchain Applications - Usage in Different Domains", Digital Object Identifier 10.1109/ACCESS.2019.2902501 VOLUME 7, 2019

3. Meghali Nandi, Rajat Kanti Bhattacharjee, Amrit Jha, Ferdous A. Barbhuiya "A secured land registration framework on Blockchain", Third ISEA Conference on Security and Privacy (ISEA-ISAP) 978-1-7281-6708-4/20 (C2020

4. Elva Leka, BesnikSelimi, and Luis Lamani "Systematic Literature Review of Blockchain Applications: Smart Contracts", IEEE International Conference on 
Madhurya J A et al., International Journal of Emerging Trends in Engineering Research, 8(9), September 2020, 5209 - 5213

Information Technologies, 978-1-7281-3274-7/19/ $\$ 31.00$ (0) 2019 IEEE

5. WeilinZheng, ZibinZheng, Xiangping Chen, Kemian Dai, Peishan Li, AndRenfei Chen "NutBaaS: A Blockchain-as-a-Service Platform" IEEE Access, Digital Object Identifier 10.1109/ACCESS. 2019.2941905 VOLUME 7, 2019

6. Jingjing Chen, TiefengCai, Wenxiu He, Lei Chen, Gang Zhao, WeiwenZou "A Blockchain-Driven Supply Chain Finance Application for Auto Retail Industry" Entropy 2020, 22, 95; DOI:10.3390/e22010095

7. Harry Halpin, Marta Piekarska "Introduction to Security and Privacy on the Blockchain", 2017 IEEE European Symposium on Security and Privacy Workshops (EuroS\&PW)

8. AshwinSekhar, RishavChatterjee, RasDwivedi, RohitNegi, Sandeep K Shukla .Entangled Blockchains in Land Registry Management.

9. Yining $\mathrm{Hu}$, MadhusankaLiyanage, AhsanManzoor, KanchanaThilakarathna, KanchanaThilakarathna, ArunaSeneviratne " Blockchain-based Smart Contracts -Applications and Challenges," arXiv:1810.04699v2 [cs.CY] 8 Jun 2019.

10. https://www.leewayhertz.com/blockchain-land-registryplatform/

11. I.Jeena Jacob, Dayanand Lal.N, Parikshith Nayaka S K , Beena G. Pillai , Nida Kouser. Ensuring Network Security using Secured Privileged Accounts. International Journal of Emerging Trends in Engineering

Research.https://doi.org/10.30534/ijeter/2020/80852020

.Volume 8 No.5, 2020 